\title{
DEDALO: Application of Structural Health Monitoring Systems on UHTC Structures
}

\author{
V. Latini ${ }^{*}, 1$, V. Striano ${ }^{2}$, F. Monteverde ${ }^{3}$, I. Rendina ${ }^{4}$ and C. Parolini ${ }^{5}$ \\ ${ }^{1}$ SAB Aerospace, Via Tiengo, 15, Benevento, 82100. Italy \\ ${ }^{2}$ Carlo Gavazzi Space, Via Gallarate, 150, 20151 Milano, Italy \\ ${ }^{3}$ National Research Council - Institute of Science and Technology for Ceramics (CNR-ISTEC), Italy \\ ${ }^{4}$ National Research Council - Institute of Microelectronics and Microsystems (CNR-IMM), Italy \\ ${ }^{5}$ Bettini Technical Division, Italy
}

\begin{abstract}
In aerospace applications the development of a reliable method of structural health monitoring (SHM) is one of the most important keys in maintaining the integrity and safety of structures, preventing catastrophic failure.

The research program DEDALO aims at developing a real size UHTC-based prototype with a complex shape equipped with a SHM system for damage detection.

A multidisciplinary approach has been adopted involving mechanical design, materials science, manufacturing processes and development of optical devices to detect strain and temperature on the as-produced UHTC articles. Former activities merged into the manufacturing of a prototype hot structure supplied with optical sensing nodes to perform a functional test at high temperature. This communication describes the preliminary findings of the project. A series of $\mathrm{ZrB}_{2}-\mathrm{SiC}$ based compositions was studied adjusting type, concentration and granulometry of reinforcing phases and additives to further identify the optimal composition for the hot structure. The pressureless sintering technique was selected privileging a near-net-shape approach to reduce the manufacturing costs.
\end{abstract}

A SHM system was developed using commercial high temperature Fiber optic Bragg Grating (FBG), for thermal monitoring, and custom silica-sapphire fiber optic strain sensor, based on Fabry-Pèrot configuration, allowing simultaneous and real time measurement of temperature and structural loads applied on the structure under investigation.

A ceramic flexible structure was developed to ease sensor installation procedure on complex shape test articles. The fiber optic sensors interrogation system was developed based on a tunable laser source.

Thermal and mechanical tests showed sensor robustness at high temperature and $0,6 \mu \varepsilon$ as accuracy on strain measurement 0 togliere up to $800^{\circ} \mathrm{C}$. Tile-shaped hot structures were manufactured, equipped with the prototype Structural Health Monitoring System (SHMS) and functionally tested at high temperature. The project will undergo a second iterative loop which foresees investigation on the final test article: $\mathrm{ZrB}_{2}-\mathrm{SiC}$ based composite hollow tip.

Keywords: Ultra-high temperature materials, SHMS, thermal protection systems, fiber optic, tip, re-entry vehicle, pressureless sintering, near-net-shape approach

\section{INTRODUCTION}

DEDALO is a research program, funded by district Lombardia, which aims at developing a prototype Ultra High Temperature Ceramic (UHTC) structure to be equipped with a Structural Health Monitoring System (SHMS). The UHTC structure represents a Thermal Protection System (TPS) for a re-entry vehicle monitored by a SHMS that should provide real time temperature and strain measurements for critical areas located on the UHTC structure in order to timely detect any structural damage.

*Address correspondence to this author at the SAB Aerospace, Via Tiengo, 15, Benevento, 82100. Italy; Tel: 00390712089501; Fax: 00390712089510; E-mail: vlatini@sabaerospace.com
The project is held by aerospace and manufacturing companies, and two research centres involved in different activities.

The project started with the development of a UHTC tile system, designed by SAB Aerospace. Subsequently the research activity has involved CNR-ISTEC for the development of the UHTC composition that meets thermomechanical requirements. Carlo Gavazzi Space in collaboration with CNR-IMM has developed the SHMS constituted by optical sensing nodes to measure both strain and temperature. The UHTC tiles, manufactured by Bettini Technical Division, have been equipped with the SHMS to perform preliminary functional tests. The project is currently approaching the final step which includes the design and 

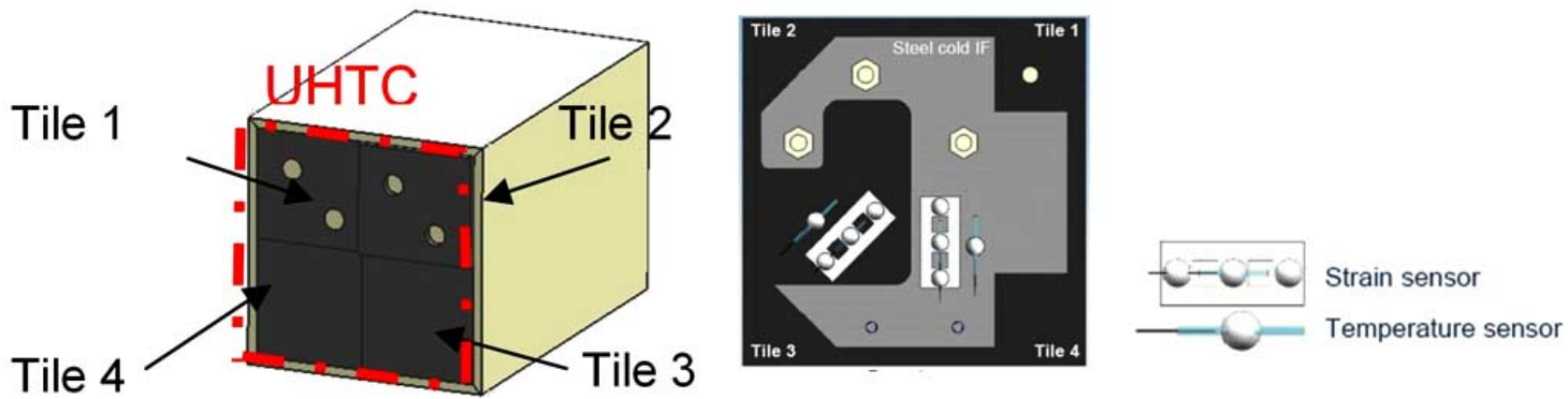

Fig. (1). Preliminary test article - UHTC tile system: (left) front side, (right) rear side with the steel c-shape plate.

manufacturing of a UHTC hollow tip equipped with optical sensors to perform functional tests at high temperature.

\section{UHTC STRUCTURE CONCEPT DESIGN}

A preliminary UHTC concept design for TPS was developed by properly placing optical sensors as shown in Fig. (1).

Fiber Optic (FO) sensors are located both on UHTC tiles and on a steel plate (gray C-shape plate in Fig. (1)) to detect strain and temperature both directly on UHTC material and on an metallic interface structure when the whole assembly is exposed to temperatures up to $800^{\circ} \mathrm{C}$. FO sensors are located on the rear side of the assembled system because they can not be directly exposed to the heat load.

The test article is composed of 4 UHTC tiles $44 \times 44 \mathrm{~mm}$ with variable thickness (5 and $8 \mathrm{~mm}$ ) and different mechanical fastening $\left(\mathrm{Al}_{2} \mathrm{O}_{3}\right.$ screws and Zirconia based glue RESBOND 904) with the steel C-shape plate which represents a sample of a re-entry vehicle cold structure.

The tile system was designed and developed to be tested at high temperature in an early stage of the project to gather information on mechanical fastening, optical sensor response and manufacturing process.

The final UHTC test article, currently under manufacturing, is a real size hollow tip screwed to complex shape connection tiles, as shown in Fig. (2).

The hollow tip (height $=125 \mathrm{~mm}$, max. diameter $=133$ $\mathrm{mm}$, wall thickness $=8 \mathrm{~mm}$ ) will be equipped with optical sensing nodes on rear side to measure both strain and temperature during functional tests at high temperature.

Taking into due account some technological constraints like a) dimensions, complexity and shape of the final test article, b) reduction of the manufacturing costs, c) feasibility of a real technological transfer at industrial level to routinely produce UHTC complex pieces, the partners of the DEDALO project selected the well-known pressureless sintering (PLS) as the best suited technique to overcome the technical and economical limitations aforementioned. This approach was also stimulated by the lack of technical attempts at industrial level that basically succedeed in fabricating real UHTC test articles by PLS.

\section{FIBER OPTIC BASED STRUCTURAL HEALTH MONITORING SYSTEM (SHMS)}

In the frame of the DEDALO research project an innovative SHM system based on FO sensors has been designed for the thermo-mechanical monitoring of Thermal Protection Systems. The system has been developed for a reliable measurement in a temperature working range of $800-$ $\sim 1000^{\circ} \mathrm{C}$.

The system is divided in two sub-systems, one for temperature monitoring and the other for quasi-static strain measurement. For thermal monitoring the temperature resistant Fiber Bragg Grating (FBG) sensors have been chosen. The FBG is an optical wavelength band pass filter realized by writing a grating inside the fiber core, periodically modulating its refractive index. The period of the refractive index is influenced by structural changes of the fiber induced by strain, temperature, or pressure to name a few.

FBGs are often used to monitor properties like strain and temperature. The amplitude of the photo-induced refractive index modulation in a grating written in a germanosilicate fiber decreases more than twofold as a result of heating to 250-500 ${ }^{\circ} \mathrm{C}$. Therefore, such gratings are unsuitable for sensors intended for use at high temperatures. Bragg gratings written in N-doped silica fibers, with an addition of fluorine in the fiber cladding, were found to be more thermostable than those written in germanosilicate ones. Its working temperature range is $-120^{\circ} \mathrm{C}-900^{\circ} \mathrm{C}$, with an accuracy of
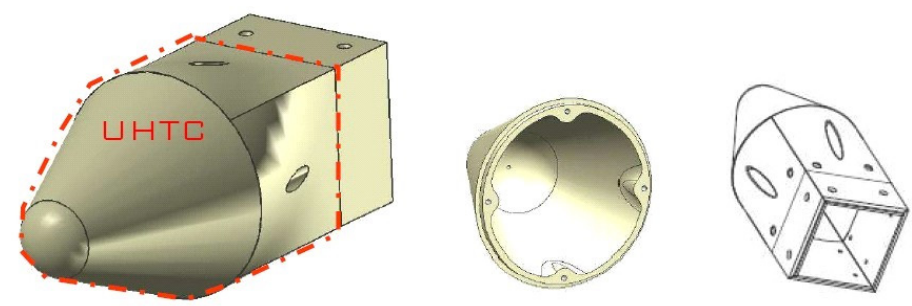

Fig. (2). final test article - UHTC hollow tip and connection tiles: (left) front side, (center and right) rear side. 
$16 \mathrm{pm} /{ }^{\circ} \mathrm{C}$ [1]. They can be exposed at temperature up to $1100^{\circ} \mathrm{C}$ for exposure time less than $10 \mathrm{~min}$.

A different approach for strain monitoring has been chosen. Commercial FO strain sensors have low reliability in high temperature range; in addition, their operation is not guaranteed for temperature up to $500^{\circ} \mathrm{C}$. To this aim a custom FO strain sensor has been designed, using single crystal sapphire fiber and Fabry-Pérot technology.

Among many fiber-optic sensing schemes, optical fiber based extrinsic Fabry-Pérot interferometric (EFPI) sensors have demonstrated their advantages such as very small size, high sensitivity and great design flexibility [2]. A fiber optic EFPI sensor can be easily constructed by inserting two fiber with their end-faces well cleaved and polished into a capillary tube. The air gap between the two fiber end-faces becomes a low finesse Fabry-Pérot cavity, which generates interference signal as a function of the cavity length and the refractive index of the medium inside the cavity. The endface of a single crystal sapphire fiber, used to generate the interferometric cavity, is characterized by a low reflectivity $(\sim 7 \%)$. This feature allows to use a two-beam interference approximation model to analyze the output interference signal $I_{O}(\lambda, d)$ from the EFPI sapphire sensor, where $d$ is the cavity length:

$$
I_{O}(\lambda, d)=I_{I}(\lambda) / 2 \cdot\left[1+\alpha(d) \cos \left(4 \pi d / \lambda+\varphi_{0}\right)\right]
$$

where, $\lambda$ is the optical wavelength, $I_{I}(\lambda)$ is the source spectrum, $\alpha(d)$ is a factor which takes into account some attenuating effects due to the geometry of the cavity and $\varphi_{0}$ is the starting phase difference between the two arms of the interferometer. It is shown in Eq. 1 that the output spectrum of the sensor is modulated by sinusoidal function due to the interference inside the cavity. Because the interferometric spectrum is function of the sensor air-gap length $d$, the demodulation of this spectral signal provides an accurate and absolute measurement of the cavity length of the EFPI sensor.

The Fabry-Pérot cavity is made by cleaving and polishing the end-faces of a standard single mode (SM) silica fiber and a $250 \mu \mathrm{m}$ sapphire fiber. For its high end- face surface the sapphire fiber has been chosen as mirror, while the SM fiber has been used as input/output beam of the interferometer.

In order to ease the installation procedure, both the fibers are aligned inside a glass capillary tube and are bonded on a

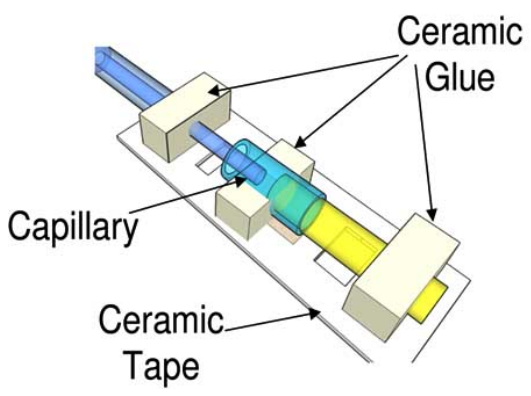

thin ceramics tape. The alignment procedure has been performed by means of a mechanical micro-manipulator. The fibers and the capillary are bonded on the tape using a low thermal expansion ceramic glue (Resbond 940LE from Cotronics), in order to prevent the fibers breaking, during the high temperature exposition, caused by the coefficient of thermal expansion (CTE) difference between the fiber and the glue. The structure of the tape has been designed in order to make mechanically independent the capillary, the SM fiber and the sapphire fiber, once bonded on the structure to be monitored. In this way, the variation of the interferometric cavity length only depends on material deformation and thermal stress of the sensor itself. The sensor layout is shown in Fig. (3).

Moreover, a structure similar to the strain sensor has been used to realize an EFPI temperature sensor. Both the fibers are bonded to the glass capillary, whose thermomechanical response has been analyzed and characterized. In this way the temperature measurement can be carried out from the measure of the capillary deformation.

For all the FO sensors, the information on the measured physical parameters is coded in the spectral response of the optical devices. Therefore, in order to extract the measurement information, an optical interrogation unit has been developed. The interrogation unit allows to perform the wavelength scanning of the reflected optical signal for both the sensors FBG and EFPI.

The FO monitoring system has been designed for the interrogation of three couples of combined sensors FBG/EFPI. The light coming from the tunable laser is coupled into a fiber optic circulator, to reduce the power losses on the arm of the reflected signal. By an 1X8 fiber optic switch, controlled by a PC, the source beam can be redirected to interrogate a single sensor. The light reflected by the selected sensor comes back into the optical circulator and is converted in a photo-current by a photodetector. Then the electrical signal is digitized by a Analog to Digital Converter (ADC) and is processed by the PC. The interrogation system is controlled by a custom Labview routine including a Matlab subroutine developed in order to analyze and decode the optical spectrum information of both the FO sensors.

All the temperature and strain sensors have been characterized by means of a comparative sensor, respectively: a $\mathrm{K}$ type thermocouple for high temperature and a resistive strain gauge for the mechanical response of

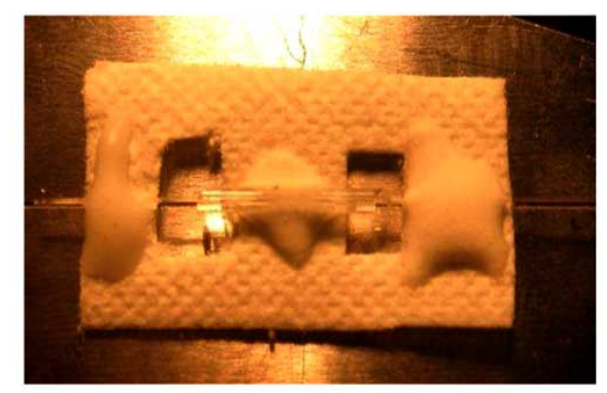

Fig. (3). EFPI strain sensor layout. 
the EFPI strain sensor at room temperature. The measured FBG sensitivity is $15 \mathrm{pm} /{ }^{\circ} \mathrm{C}$, comparable with the technical datasheet $\left(16 \mathrm{pm} /{ }^{\circ} \mathrm{C}\right)$. The CTE of the EFPI temperature sensor has been measured, obtaining a mean value of $2.4 \pm$ $0.2 \mu \varepsilon /{ }^{\circ} \mathrm{C}$, on the temperature range $25^{\circ} \mathrm{C}-400^{\circ} \mathrm{C}$. The mechanical response at room temperature of the EFPI strain sensor has been measured and compared with the Strain gauge. The ratio between their strain measurement is $1,016 \pm$ 0,002 .

Moreover, for the thermo-mechanical behavior of the EFPI strain sensor, a numeric model has been developed and the transient response of the sensor has been measured at different temperature in the range $100^{\circ}-700^{\circ} \mathrm{C}$. Comparing the numerical model and the transient response measurements, the $\langle\mathrm{CTE}>$ of the sensor have been carried out. The measured value is: $1,9 \pm 0.2 \mu \varepsilon /{ }^{\circ} \mathrm{C}$.

\section{DEVELOPMENT OF UHTC COMPOSITIONS}

Four different compositions based on $\mathrm{ZrB}_{2}-\mathrm{SiC}$ were produced, by adjusting not only type and granulometry of the reinforcing $\mathrm{SiC}$ phase but also adding specific ceramic sintering aids.

The starting formulations (stoichiometry not reported) were mixed using de-mineralized water and $\mathrm{SiC}$ milling media in rotating tephlon lined mills for several hours, and then dried through well-established atomization procedure. Specific additives (organic binders and deflocculants) were also added to the starting formulations in proper amounts to improve dispersability of the ceramic powders during mixing, flowability of the atomized products and compactness of the cold isostatically pressed pieces. Previous studies reported results on the optimization of powder processing [3] or sintering of $\mathrm{ZrB}_{2}-\mathrm{SiC}$ by PLS [4] at laboratory scale.

The set-up of optimal sintering conditions was done by using cold-isostatically pressed discs and various sintering cycles using graphite heating element furnace in inert atmosphere. The first part of the heating cycle included the debonding stage (to remove all the organic additives added), followed by the hot-consolidation run characterized by specific heating/cooling rates, intermediate holds and

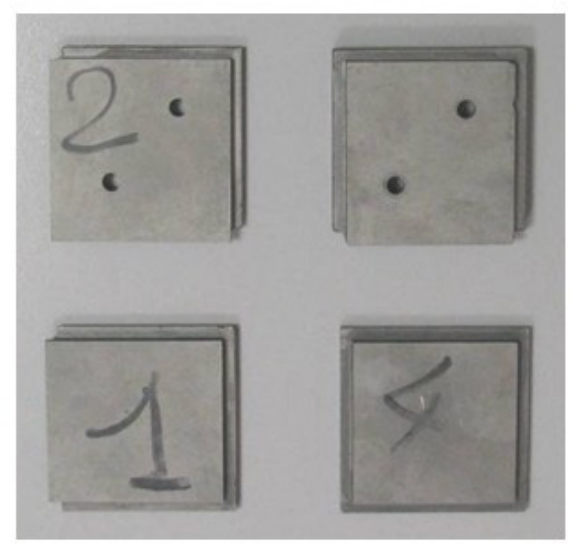

maximun set-points (up to $2150^{\circ} \mathrm{C}$ ). Depending on the composition used, various sintering conditions were optimized.

The preliminary checks on the as-sintered materials consisted of: measurements of the linear shrinkage and relative density (Archimede's method), X-ray diffraction, and scanning electron microscopy. Typical values of the relative densities approached $95 \%$.

The following technological step was to realize UHTC tiles $44 \times 44 \times 5 \mathrm{~mm}$, by testing all the four compositions. For each of them, a "green" piece of suitable initial dimensions was cold-isostatically pressed, and then dry machined/drilled through mechanical tools. The primary aim was to shape properly the UHTC article before processing it by PLS. Some attempts are shown in Fig. (4). At the end of this trade-off phase, a composition based on $\mathrm{ZrB}_{2}-\mathrm{SiC}$ was selected. A new powder mixture batch of about $150 \mathrm{~kg}$ was prepared, according to the procedures aforementioned.

\section{POWDER PROCESSING, MANUFACTURING AND CHARACTERIZATION}

The raw materials $\mathrm{ZrB}_{2}$ and $\mathrm{SiC}$ were introduced in a ball milling machine with water for about 10 hours in order to obtain an homogeneous slurry. The slurry was first spraydried in an atomizer, and then cold isostatically pressed to produce "green" cylinders. The massive cylinders were sliced and ground obtain "green" pieces of the required shapes with overmaterial to prevent that possible distortions from the expected shape, during sintering process, would cause non conformity of the final dimensions.

The "green" pieces were debonded in an oven with inert atmosphere and pressureless sintered in a graphite furnace at a temperature over $2000^{\circ} \mathrm{C}$ in argon atmosphere. "Ad hoc" thermal cycles were tuned in order to guarantee a slow and homogeneous heating over all the parts of the tiles to prevent fractures in the bulk of the material: the main problem was connected with the presence of holes, non uniform thickness and parts of the tiles not lying on a flat surface.

The sintered pieces were first cut with Electro Discharge Machining and then properly machined.

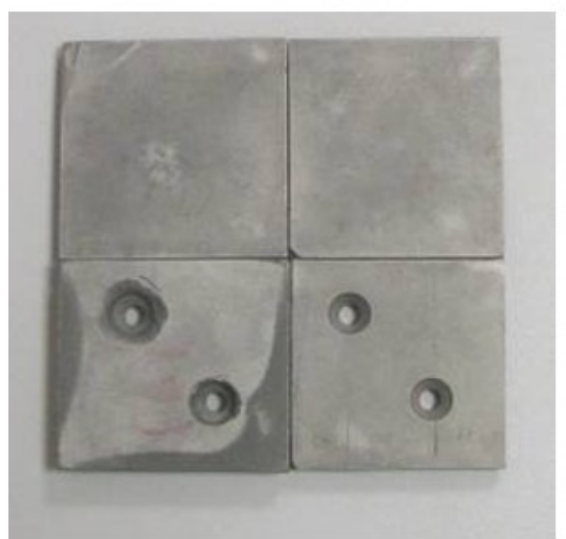

Fig. (4). UHTC tiles. 
In order to protect and insulate optical fibers along their path from the UHTC tiles to the SHMS interrogation system, $\mathrm{Al}_{2} \mathrm{O}_{3}$ insulating tiles were manufactured to be screwed to an AISI 316L supporting frame representative of a metallic interface structure. The whole structure, assembled for the test campaign is described in the next section.

Parallel to the manufacturing set-up, the base material was fully characterized from the microstructural and thermomechanical point of view. In the following some determinations are described.

\subsection{Microstructure}

In contrast to the results obtained during the trade-off phase, the transition to a preparation at industrial level introduced unexpected modifications in the final chemistry of the atomized product. Such chemical changes, mainly the superior residual content of oxygen, caused a less pronounced sinterability that led to produce sintered compact characterized by final relative densities below $85 \%$. The SEM micrograph in Fig. (5) shows the typical distribution of the residual porosity.

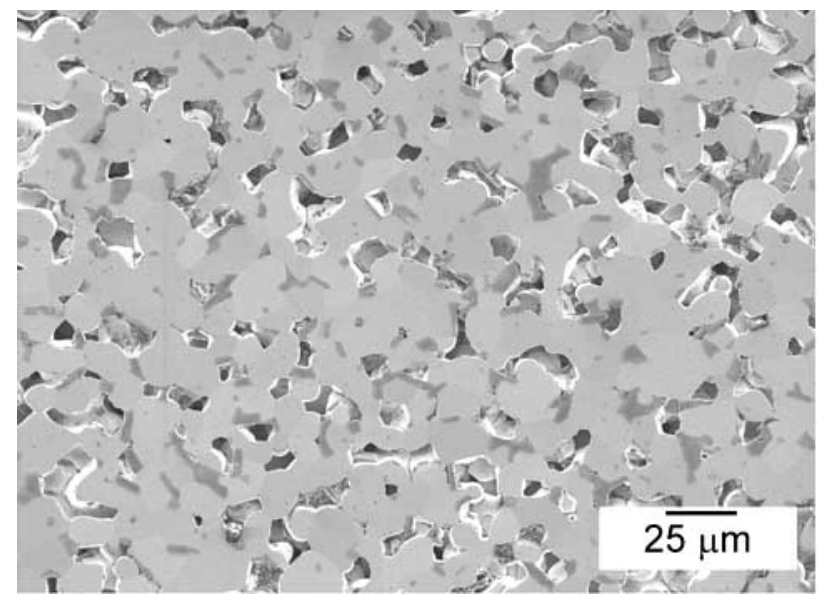

Fig. (5). SEM micrograph: polished section.

\subsection{Mechanical Properties}

The flexural strength was determined at room temperature and at $1500^{\circ} \mathrm{C}$ (in air) in a 4-pt configuration using $45 \times 4 \times 3 \mathrm{~mm}$ chamfered bars. Fracture toughness was determined according to the "chevron notched beam" method using $45 \times 4 \times 3 \mathrm{~mm}$ bars (see Table 1).

Table 1. Flexural Strength and Fracture Toughness

\begin{tabular}{|c|c|c|}
\hline \multicolumn{2}{|c|}{ Flexural Strength, MPa } & Fracture Toughness, MPa $\sqrt{ } \mathbf{m}$ \\
\hline \hline $25^{\circ} \mathrm{C}$ & $1500^{\circ} \mathrm{C}$ & $25^{\circ} \mathrm{C}$ \\
\hline $183 \pm 16 \mathrm{MPa}$ & $107 \pm 8 \mathrm{MPa}$ & $2.5 \pm 0.3$ \\
\hline
\end{tabular}

\subsection{Resistance to Oxidation}

This characteristic was evaluated in an "arc-jet" gallery (available at the Department of Aerospace Engineering, University of Naples "Federico II, Italy) in order to assess the suitability of the as-produced material in simulated reentry conditions. "Ad-hoc" articles with hemispherical shape were machined by EDM and exposed to an aeroheating environment in high enthalpy flow (Fig. 6).

The sample, $5 \mathrm{~mm}$ radius of curvature, reached and maintained a surface temperature of $2100 \mathrm{~K}$ for $1 \mathrm{~min}$. The visual appearance after testing (Fig. 7) verified that the sample experienced minimal damage on surface.

After the manufacturing of the UHTC tiles (shown in Fig. 4), the final test article, a complex shape one, was produced with the same composition: a hollow tip, shown in Fig. (8), and four connection tiles to join the tip to the steel frame.

The sintering process required specific "ad hoc" solutions: a "green" support of the same material of the tip was used and cut in two parts for the manufacturing of the tip to prevent the formation of cracks caused by friction with the graphite plates. A thin layer of an inert powder mixture was positioned between the support and the tip to prevent adhesion.

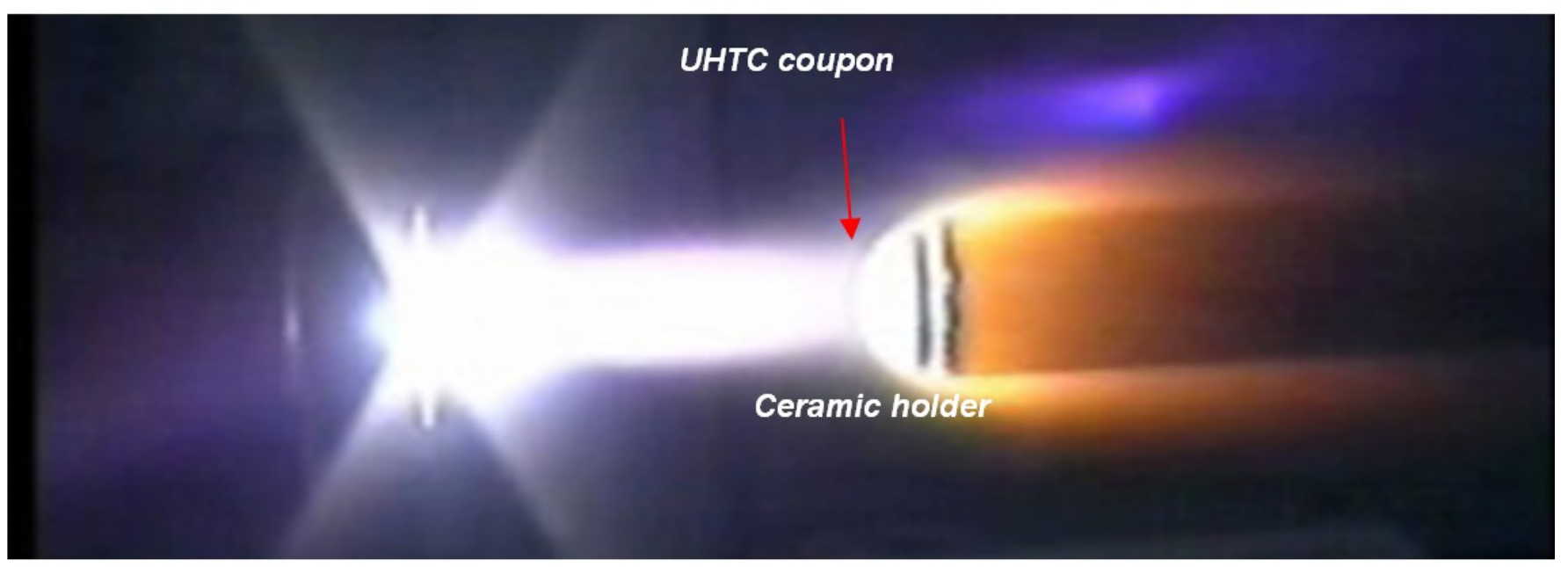

Fig. (6). Experimental set-up during exposure to the hot reactive gas stream. 

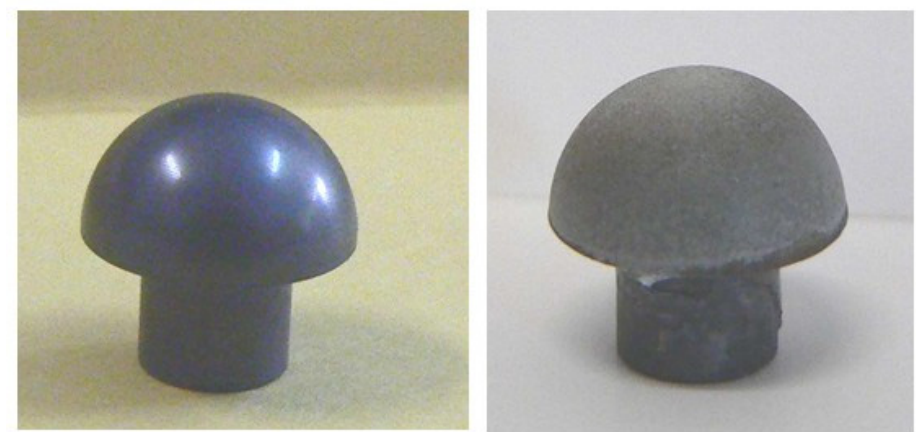

Fig. (7). Hemispherical sample before (left) and after testing (right).
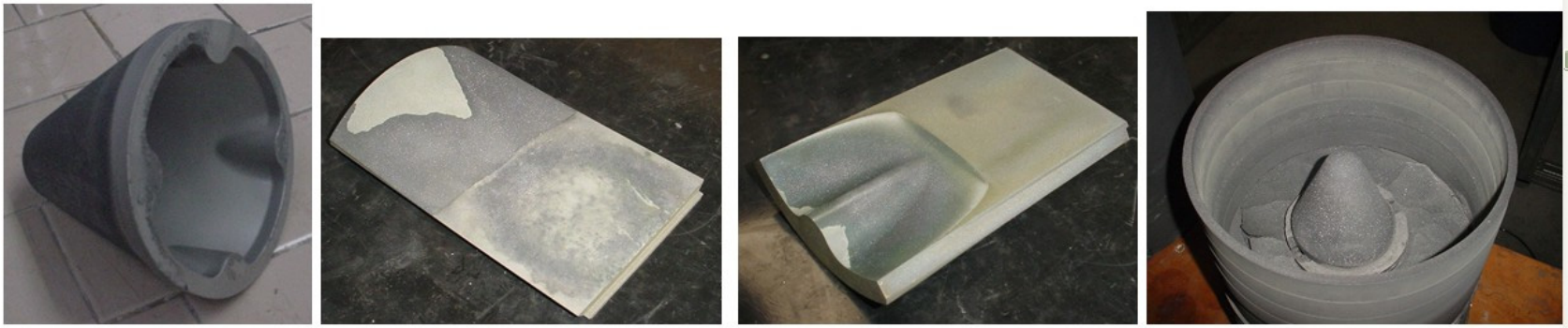

Fig. (8). (left) UHTC hollow tip, (centre) UHTC connection tiles, (right) 'green' support.

The sintered pieces were first cut with Electro Discharge Machining and then properly machined.

\section{TEST}

Preliminary functional test aimed to the test UHTC hot structure, equipped with the SHMS, at temperatures up to $850^{\circ} \mathrm{C}$.

The test facility is a Carbolite Tersid air furnace, as shown in Fig. (9). Once the OF sensors are located behind the UHTC tiles, and the whole mechanical system is insulated on lateral sides by $\mathrm{Al}_{2} \mathrm{O}_{3}$ tiles, the structure is inserted in the tubular air furnace and exposed to high temperature. FO sensors measure strain and temperature in real time for the whole duration of test.

\subsection{Test Configuration and Conditions}

Test configuration is shown in Fig. (10).

UHTC tiles are fastened (tiles \#1 and \#2 by $\mathrm{M} 4 \mathrm{Al}_{2} \mathrm{O}_{3}$ screws, tiles \#3 and \#4 by Zirconia-based glue) to the $\mathrm{C}$ shape steel plate representing the cold interface structure.
The C-shape plate is then screwed to the AISI 316L supporting frame which is insulated by $20 \mathrm{Al}_{2} \mathrm{O}_{3}$ tiles. Four fiber optic sensors (2 temperature sensors and 2 strain sensors) are located behind UHTC tiles and the C-shape steel plate, as shown in Fig. (13). Two fiber optic sensors (1 temperature sensor and 1 strain sensor) are located behind a $\mathrm{Al}_{2} \mathrm{O}_{3}$ tile, as shown in Fig. (13). Optical fibers run inside a protective pipe inside the supporting frame in the path from the UHTC tiles toward the interrogation system located outside the test furnace. Four K-type thermocouples are located inside the structure: two for comparison with FO temperature sensor results, and two to measure the furnace temperature.

The furnace set up is shown in Fig. (11): entrance area is kept OFF (furnace cold side) while central and remaining areas (hot side) are set to $1000^{\circ} \mathrm{C}\left(700^{\circ} \mathrm{C} / \mathrm{hr}\right.$ heating rate). The test article, as shown in Fig. (11), reaches the furnace central area exposing UHTC tiles to maximum temperature gradient between furnace hot and cold sides. The furnace temperature is measured by the use of K-type thermocouples ( $\mathrm{T}_{\text {mid }}$ and $\mathrm{T}_{\text {ext }}$, as shown in).

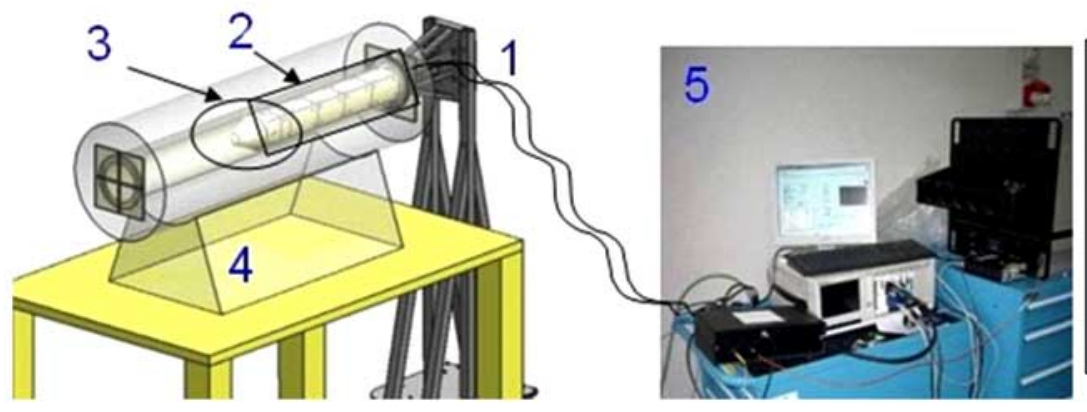

1) AISI $316 \mathrm{~L}$ supporting frame + C-shape steel plate

2) $\mathrm{Al}_{2} \mathrm{O}_{3}$ insulating tiles

3) UHTC test article

4) Furnace

5) Interrogation system

Fig. (9). Test facility. 


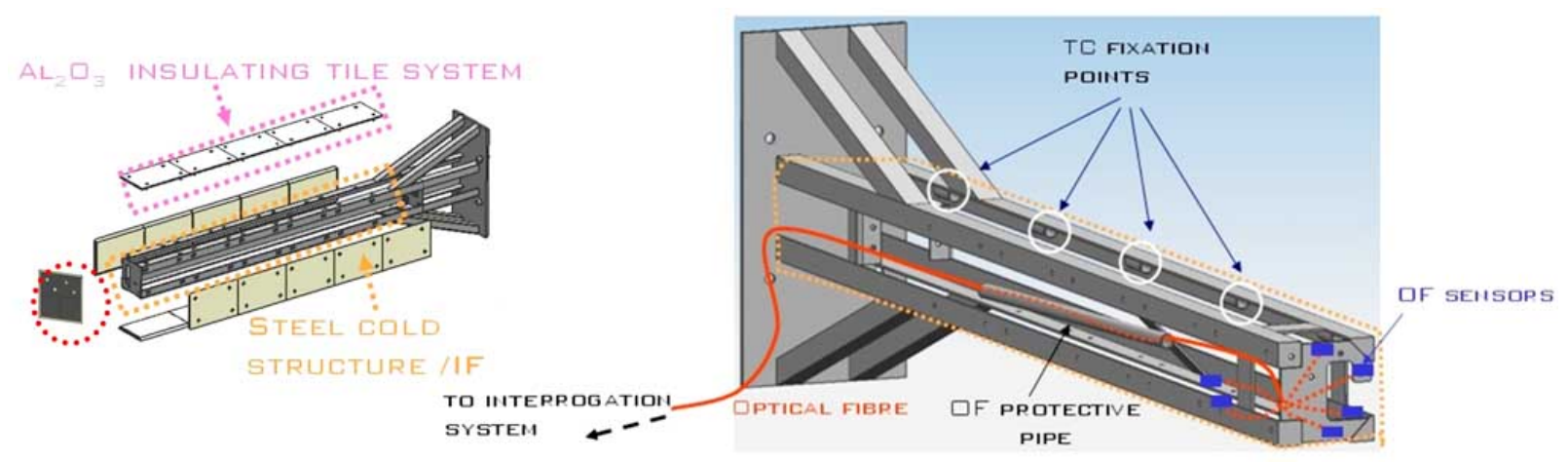

Fig. (10). Test configuration.
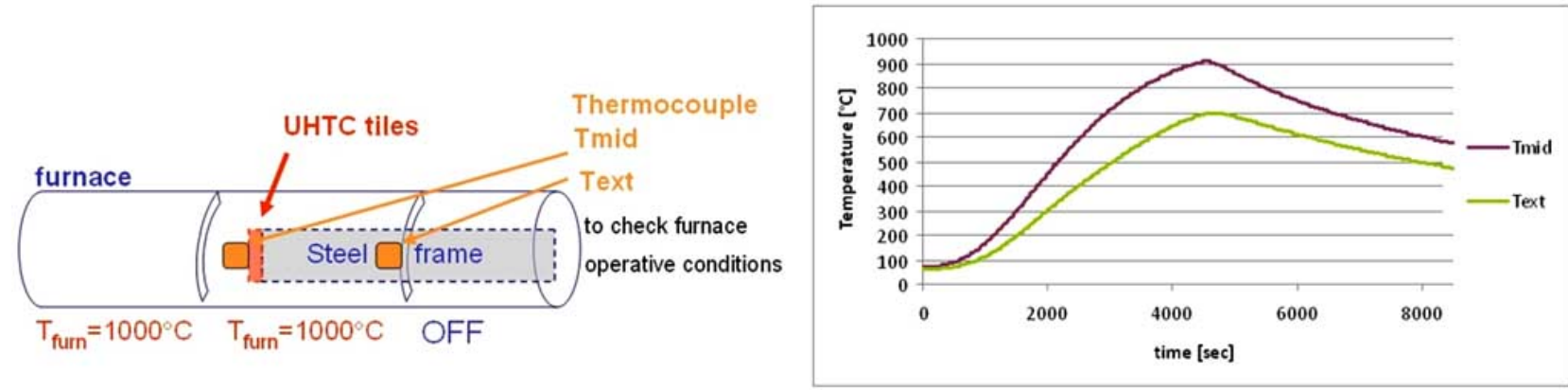

Fig. (11). (left) furnace set up; (right) test conditions.

\subsection{Installation}

FO sensor have been first installed on UHTC and $\mathrm{Al}_{2} \mathrm{O}_{3}$ tiles (as shown in Fig. 13), then FO wires have been routed inside the protective pipe and outside the supporting structure. At last all the ceramic tiles have been fastened to the supporting structure and placed inside the furnace for curing time (Fig. 14).

In order to match the CTE of the FO sensors and the monitored materials, several thin layers of different ceramic glues (RESBOND 919 and RESBOND 904 ) have been spread on the region of the installation, as shown in Fig.

(12). The CTE of the glue layers increases from the material to the sensor. For each spreading step, the glue has been cured as indicated by the manufacturer.

\subsection{Test Results}

FO strain sensors results are shown in Fig. (15).

Strain sensor located on the C-shape steel plate, behind UHTC tile, shows, as expected, maximum deformation $5000 \mu \varepsilon\left(\mathrm{T}_{\text {furnace }}=830^{\circ} \mathrm{C}\right)$, higher than the ones detected on ceramic tiles. In section 2 and 4 of the curve in Fig. (15), the slope of steel strain sensor detects almost the same value for

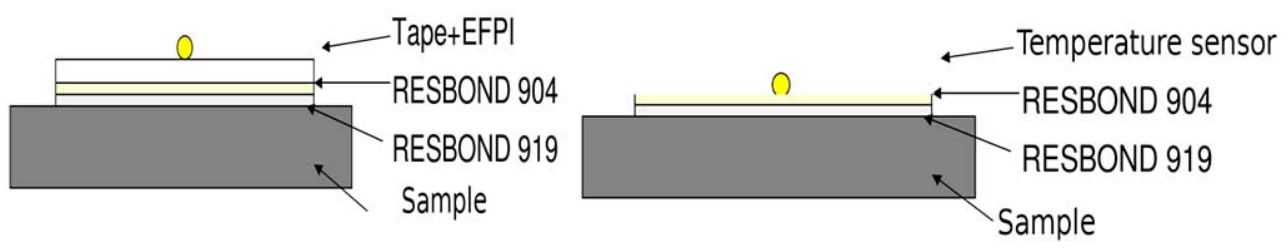

Fig. (12). Configuration of the glue layers.

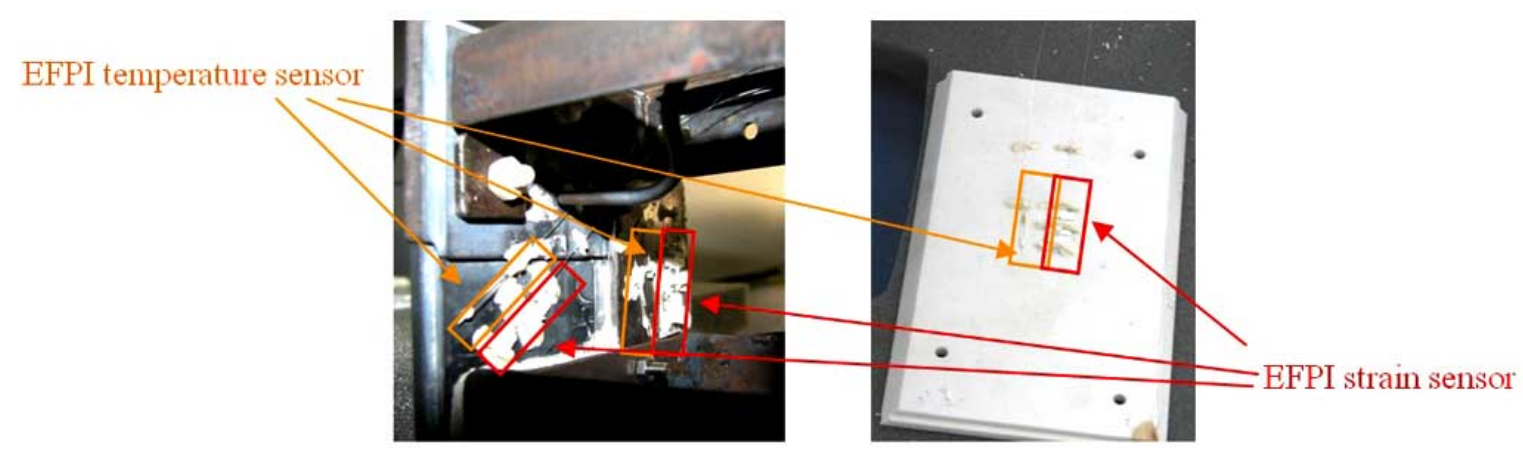

Fig. (13). (left) FO sensor installation on UHTC tiles; (right) FO sensor installation on $\mathrm{Al}_{2} \mathrm{O}_{3}$ tiles. 

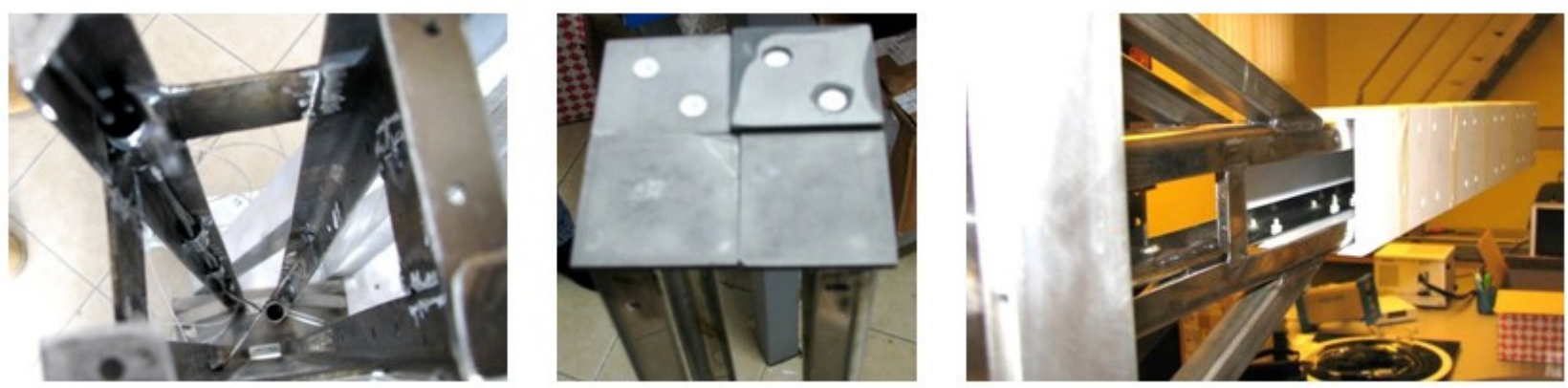

Fig. (14). (left) AISI 316L frame; (centre) UHTC tile installation; (right) $\mathrm{Al}_{2} \mathrm{O}_{3}$ tile installation.

$\mathrm{CTE}_{\text {steel }}=15 \mu \varepsilon /{ }^{\circ} \mathrm{C}$ which can be compared with values in literature.

In section 3 a relaxation of the material is shown, not detected during test at high temperature on single steel sample: this is probably due to the relaxation of some mechanical constraints on the C-shape steel plate performed by surrounding tiles and the AISI $316 \mathrm{~L}$ supporting structure.

Strain sensor on $\mathrm{Al}_{2} \mathrm{O}_{3}$ tile detects a deformation lower than that on the UHTC tile because the $\mathrm{Al}_{2} \mathrm{O}_{3}$ tile is located near the exit side of the furnace (the one switched OFF), therefore a lower heat load is applied.

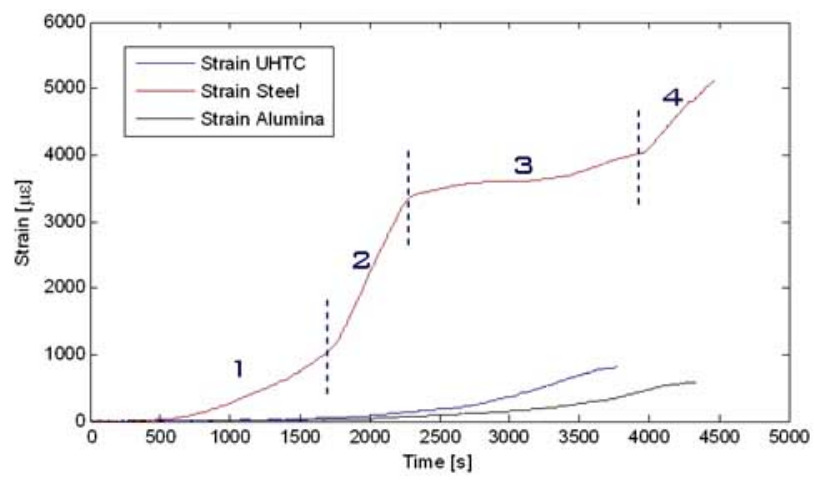

Fig. (15). FO strain sensors results.

Strain sensor on UHTC tile detects a maximum deformation $\sim 750 \mu \varepsilon @ / 700^{\circ} \mathrm{C}$ lower than the expected value measured in preliminary tests $(3500 \mu \varepsilon)$. UHTC deformation graph (Fig. 16) shows a discontinuous shape due to the alternation of expansion and shrinkage detected during the test. The global effect is a progressive expansion of the material repeatedly reduced by small amplitude shrinkage waves.
The post-test inspection showed the sensor was still completely attached to the UHTC surface. For this reason former behaviour, not detected during test at high temperature on single ceramic tile, can not be caused by sensor detachment from the tile surface.

This behaviour might have been caused by the mechanical coupling of the whole structure: each UHTC tile is surrounded by ceramic tiles under the same heat load conditions. Therefore the thermal expansion of each tile is blocked by the expansion of surrounding tiles. The continuous attempt to expand and the subsequent block, might generate former waving behaviour.

FO temperature sensors results are shown in Fig. (17) and compared with thermocouple results.

TC4 detects temperature inside the furnace (in the middle area) but outside the test article.

TC1 and 2 are located behind the UHTC tiles, inside the test article.

TC3 is located near the $\mathrm{Al}_{2} \mathrm{O}_{3}$ tile where $\mathrm{FO}$ sensors are installed.

FO temperature sensors show reliable results, when compared with the thermocouple ones, only up to $200^{\circ} \mathrm{C}$. For higher temperatures the thermal gradient between FO sensor and thermocouple reaches $50 \div 60^{\circ} \mathrm{C}$. This is due to the melting of the borosilicate capillary tube which, in the temperature sensor construction, is the probe itself.

The melting effect is less critical on the FO deformation sensor because the capillary tube is used only as a mechanical constraint to guarantee fiber alignment. The material softening, prior to melting process, does not affect deformation measurements.
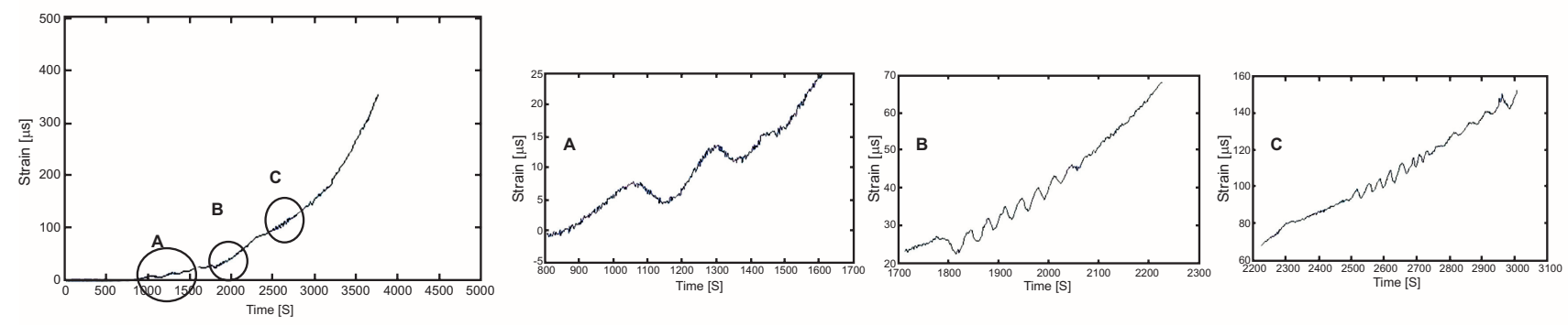

Fig. (16). Details of FO strain sensor on UHTC tile results. 


\section{CONCLUSIONS}

A functional test of SHMS installed on a UHTC TPS has been performed successfully during the first part of DEDALO research project.

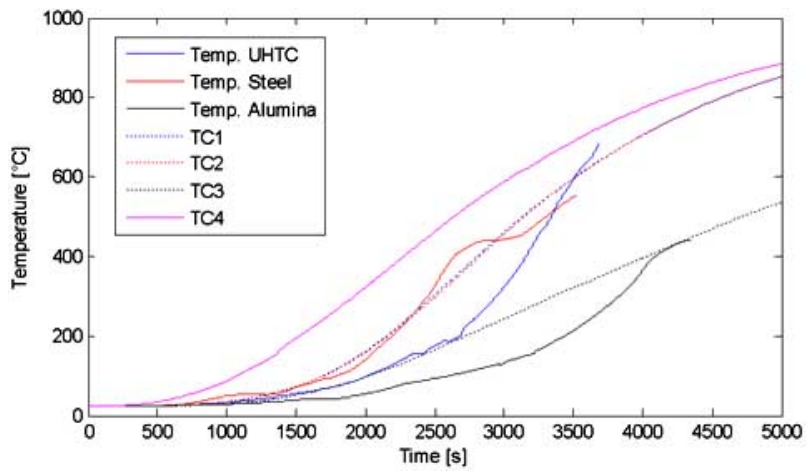

Fig. (17). FO temperature sensors results.

Test results confirm manufacturing process feasibility together with the identification of the suitable UHTC composition for the production of complex shape hot structures. Further optimization of the powder processing is necessary to limit oxygen contamination in order to provide better sinterability and therefore mechanical robustness to the sintered articles.

Regarding the SHMS a critical feature has been detected on the use of borosilicate capillary tube for FO temperature sensor to be substituted with quartz new ones.

Former solutions will be implemented in the last part of the project to be tested on a UHTC hollow tip.

\section{REFERENCES}

[1] O.V. Butov, K.M. Golant, and I.V. Nikolin, "Ultra-thermo-resistant Bragg gratings written in nitrogen-doped silica fibers", Electron. Lett., vol. 38, no. 11, pp. 523-525, 2002.

[2] H. Xiao, J. Deng, G. Pickrell, R. G. May, and A. Wang, "Singlecrystal sapphire fiber-based strain sensor for high-temperature applications", J. Lightwave Technol., vol. 21, no. 10, pp. 22762283, 2003.

[3] T. Huang, G. E. Hilmas, W. G. Fahrenholtz, and M. C. Leu, "Dispersion of zirconium diboride in an aqueous, high-solids paste", Int. J. Appl. Ceram. Technol., vol. 4, no. 5, pp. 470-479, 2007.

[4] W.G. Fahrenholtz, G.E. Hilmas, S.C. Zhang, and S. Zhu, "Pressureless sintering of zirconium diboride: particle size and additive effects", J. Am. Ceram. Soc., vol. 91, no. 5, pp. 1398-1404, 2008. 